Article

\title{
Effects of the Geometric Parameters of Mixer on the Mixing Process of Foam Concrete Mixture and Its Energy Efficiency
}

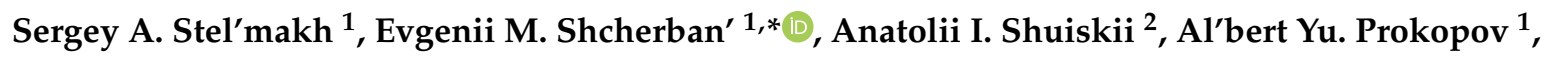 \\ Sergey M. Madatyan ${ }^{2}$, Ivan A. Parinov ${ }^{3}$ and Alexander V. Cherpakov ${ }^{3,4}$ (i) \\ 1 Department of Engineering Geology, Bases and Foundations, Don State Technical University, \\ 344000 Rostov-on-Don, Russia; sergej.stelmax@mail.ru (S.A.S.); prokopov72@rambler.ru (A.Y.P.) \\ 2 Department of Technological Engineering and Expertise in the Construction Industry, Don State Technical \\ University, 344000 Rostov-on-Don, Russia; a2293613@mail.ru (A.I.S.); mcm76@mail.ru (S.M.M.) \\ 3 I. I. Vorovich Mathematics, Mechanics, and Computer Science Institute, Southern Federal University, \\ 344090 Rostov-on-Don, Russia; parinov_ia@mail.ru (I.A.P.); alex837@yandex.ru (A.V.C.) \\ 4 Department of Information Systems in Construction, Don State Technical University, \\ 344000 Rostov-on-Don, Russia \\ * Correspondence: au-geen@mail.ru
}

Received: 27 September 2020; Accepted: 10 November 2020; Published: 13 November 2020

\begin{abstract}
The paper studies the influence of the geometric parameters of the mixer on the mixing process, the construction of the mixing body, its location in the mixer bulk, and the mixer shape and geometry. The technique of calculating the power spent on mixing the foam concrete mixture is described. The effects of the ratio of the mixture height to the mixer diameter, the number and width of reflective partitions, and the shape of the conical part of the mixer on the homogeneity of the foam concrete mixture and the power consumption are considered. The optimal ratios of the foam concrete mixture height to the mixer diameter have been determined. Moreover, the optimal range of the ratios of the partition width to the mixer diameter has been established, in order to obtain a homogeneous foam concrete mixture throughout the volume with lower energy consumption. The optimal values of the angle of the mixer conical part for the preparation of a foam concrete mixture have been determined.
\end{abstract}

Keywords: foam concrete mixer; foam concrete mixture; geometric parameters; mixing process; mixture homogeneity; power consumption; power factor

\section{Introduction}

Local materials and industrial waste are widely used in the manufacture of foam concrete goods. They have an unstable chemical and mineral composition and dispersion, which impede the production of foam concrete with stable and improved quality indicators. The simplicity and availability of technology and local raw materials make foam concrete a relatively cheap and in-demand material for construction.

The main shortcomings in the manufacture and use of non-autoclave-aerated concrete are as follows:

(i) the lack of reasonable recommendations that allow predicting the quality of goods by changing the mixer type, since the available recommendations are of a private nature with a low degree of generalization; 
(ii) the unreliability of equipment used due to defects in individual units (blades, hoses, valves, receivers, gearboxes, and so on);

(iii) failure to meet the requirements for noise, vibration, beating, dust emission, electrical safety, ease of control, etc.;

(iv) heterogeneity of the resulting material in the terms of the quality of macrostructure, density, and strength (unacceptably high coefficients of variation).

The mixing process is also influenced by the geometric parameters of mixer, the construction of mixing body, its location in the mixer bulk, the mixer shape and geometry, the ratio of the activator sizes to the mixer, and the shape and dimensions of mixer blades.

In the petrochemical technology, sufficient experience has already been accumulated in modeling, calculation, design, and practical use of such mixing equipment. However, similar studies in the construction industry are insufficient and require additional consideration [1-3]. Analysis of existing mixer designs used for the production of building materials and in petrochemical engineering $[1,2]$ showed a wide variety of mixer and activator types. At the same time, the performance characteristics were approximately the same.

In addition to the mixing energy applied to the fresh concrete (i.e., shearing during mixing), the shear history after mixing is also important. This applies especially to binder-rich concretes such as the different types of high-performance concrete (HPC). With this in mind, the shear rate is analyzed inside the drum of a concrete truck mixer. The objective is to better understand the effect of transport of fresh concrete, from the plant preparing mixture to the building site. The analysis reveals the effects of different drum loading bulk and its rotational speed. Moreover, the effects of yield stress and plastic viscosity are investigated. The studies show that the shear rate decreases in an exponential dependence with increasing drum loading bulk. It is also shown that, for a given drum speed, the shear rate decreases with increasing both the plastic viscosity and the yield stress [4].

Flexural tests were performed to investigate the influence of mixing time and mixer type on the performance. It can be concluded that mixing is beneficial for flat-type fibers in a fiber-reinforced concrete, but the performance of crimped or embossed fibers is equal. Moreover, longer mixing times ( $>10 \mathrm{~min}$ ) in a pan mixer are detrimental to the performance, whereas the performance in a tilting drum mixer does not change even after a mixing time of $60 \mathrm{~min}[5,6]$.

The optimization of a two-star planetary concrete mixer in terms of the wear resistance of blades was proposed, and a new design of the shape of mixing blades was shown and discussed. The authors proposed a new validation procedure of the mixing blades in the terms of wear resistance and efficiency, using three-dimensional (3D) optical scanners. This new wear measuring procedure is classifiable as a digital wear evaluation method, included in the field of digital tribology. The results demonstrate that the proposed new blade geometry improves the wear resistance, extends the useful life, and enhances the discharge operation $[7,8]$.

A coaxial mixer consisting of an anchor and Rushton turbine was considered in [9,10]. Its solid suspension characteristics were studied with the help of computational fluid dynamics (CFDs). The just-suspension speed of the impeller was predicted on the basis of the Eulerian-Eulerian method and modified Brucato drag model. The results showed that the coaxial mixer operating under co-rotation mode should have the best performance, and a faster anchor speed was beneficial to the solid suspension, enhancing the turbulent intensity at the bottom. At the same time, the inner Rushton turbine played a dominant role in the solid suspension due to its high rotational speed. However, an extremely high inner impeller speed would worsen the uniformity of solid distributions. Moreover, the results revealed that a higher overall solid volume fraction and a smaller Shields number worsened the performance of the solid suspension. Moreover, the solid suspension was more susceptible to solid density compared with particle diameter at the same Shields number gradient.

The authors of [11] discussed the performance of three different static mixers in terms of pressure drop generated by both single-phase flow and liquid-liquid flow in a turbulent flow regime and in terms of emulsification performance. The three motionless mixers compared were the well-known 
$\mathrm{SMX}^{\mathrm{TM}}$ and SMV ${ }^{\mathrm{TM}}$ and the new version of SMX called SMXPlus ${ }^{\mathrm{TM}}$. This experimental study aimed at highlighting the influence of dispersed phase concentration and some geometrical parameters such as the number of elements and the design of the motionless mixer on droplet size distribution characteristics. Lastly, experimental results were correlated in terms of Sauter mean diameter as a function of hydrodynamic dimensionless numbers. The authors of [12] compared the performance of the new mixer with the Kenics, SMX, and Komax static mixers. The pressure drop ratio (Z-factor), coefficient of variation $(\mathrm{CoV})$, and extensional efficiency $(\alpha)$ were used to evaluate power consumption, distributive mixing, and dispersive mixing performances, respectively, in all mixers. The new mixer showed a superior mixing performance compared to the other mixers.

The authors of [13] described the twin-screw conical mixer widely used in industries for its high mixing rate and quality. The effects of rotation speed and sweeping speed on mixing rate and quality were studied. In order to reveal the mixing performance of this mixer, the particle size ratio was investigated. The simulation results indicated that particles were well mixed in the overall and local mixing domain, as well as in the binary system, with a large particle diameter ratio of 1:3. The mixer improved mixing performance at a specific rotation ratio. By considering both the mixing performance and the power consumption, the optimal condition for operation was obtained. Compared with other parts of the mixer, the wear rate on the screw edge was faster [13].

The terms mixers and agitators are often used interchangeably. Mixers are devices that blend combinations of liquids and solids into a homogeneous product. Agitators provide a mechanical action to keep dissolved or suspended solids in solution. Both operate on basically the same principles, but the variations in design, operating speed, and applications divide the actual function of these devices. There are two primary types of mixers: propeller/paddle and screw (batch and mixer-extruder types). The product quality and brake horsepower are the parameters that can be used to indirectly gauge the performance of a mixer. There are only three major operating concerns for mixers: set-up, incoming feed rate, and product viscosity [14].

The discrete element method (DEM) was used to simulate the mixing of multiple components contained in a typical commercial whey protein mixture [15]. An effective non-sampling mixing index or the subdomain-based mixing index (SMI) was introduced to assess the mixing levels. A half-filled cylindrical double ribbon mixer was simulated, and the values of SMI were obtained for the following cases: (i) rotating ribbon, (ii) rotating cylinder, (iii) rotating cylinder with a static ribbon, and (iv) rotating cylinder and ribbon. The simulation results found that the SMI values ranged from 0 (segregation condition) to $0.91-0.94$ (fully randomly mixed condition) within a time range of 0-60 s [15].

Turbulent mixers are of particular interest, since it was established [3] that, during the formation of structures of building materials during turbulent mixing, the smallest cement particles are the first to move to extended interfaces, where they rapidly hydrate and form high-strength cement stone compounds. In this case, the type of mixing element was chosen taking into account the viscosity of the medium being mixed, since this viscosity determined the forces tending to extinguish the flow in the mixed system.

To study the composition-structure-properties dependences, modern methods of research of building materials were used. For this purpose, thermal analysis, $X$-ray phase analysis, and optical and electron microscopy were used. The use of innovative research methods [16-18] allowed for a more detailed assessment of the distribution of the properties of the finished product material throughout the entire bulk of goods.

Figure 1 shows the recommended mixture viscosity limits for the most common types of activators. On the basis of results of the analysis of the main types of mixing devices (anchor, propeller, blade, turbine, frame, screw, belt) for the preparation of foam concrete mixtures in a turbulent mode, the following factors were found:

(i) the viscosity of the mixture varies in a wide range; therefore, vane activators, the viscosity range of which is $10^{-3}$ to $10^{1.5}$, are more suitable for the studied mixtures; 
(ii) for quick adjustment of the speed mode of activator, the most acceptable is the paddle activator, capable of operating in a wider range of speeds;

(iii) analysis of the design features of activators shows that the process of manufacturing paddle activators is simpler, more technological, faster, and more economical.

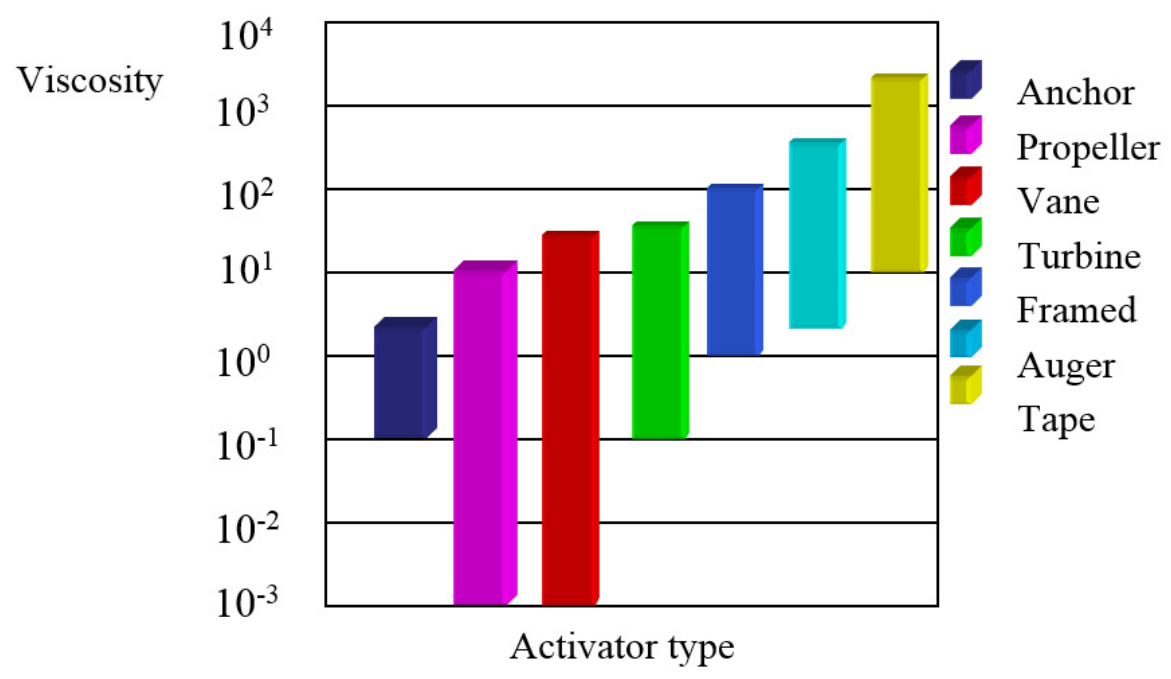

Figure 1. Selection of the type of activator depending on the viscosity of the stirred medium.

From the given data, it follows that the blade type of the activator is most optimal for the preparation of foam concrete mixtures with a density from $250 \mathrm{~kg} / \mathrm{cm}^{3}$ to $1100 \mathrm{~kg} / \mathrm{cm}^{3}$.

\section{Objects and Methods of Research}

Recently, a large number of new mixer designs were developed [1] for the preparation of aerated concrete mixtures. The implementation of a stable mixing process is associated with significant difficulties and achieved mainly empirically for a specific type of mixer. The composition and volume of mixed materials are selected practically without a reliable degree of generalization [19].

In the laboratory of the Department of Technological Engineering and Expertise in the Construction Industry, Don State Technical University, an experimental study was carried out on the optimization of the geometric parameters of mixer and activator to obtain a high-qualitative foam concrete mixture. The composition of the foam concrete mixture was selected on the basis of the required density of the finished foam concrete (Table 1). We used Portland cement 500 (PC 500), two types of foaming agent (Penostrom (FA1) and Morpen (FA2)), and sand with a fineness modulus of $M_{c r}=1.15$.

The average density of foam concrete was determined by testing samples dried to constant weight in accordance with Russian GOST 25485-2019. Samples were made from the working composition in batches of 9-12 pieces and were tested in three pieces [20]. Determination of the properties of raw materials was carried out according to generally accepted methods in accordance with the current regulatory documents [21].

The original type of mixer was chosen according to its design. Two six-blade turbines allow efficient mixing over a wide range of mixture viscosities. The mixer has a cylindrical shape, which is most suitable for the manufacture of foam concrete mixture. Moreover, the design of this mixer allows one to vary the number of partitions, types of mixers, and combination of turbine types. It allows one to more fully assess the influence of the geometry and type of the construction of the foam concrete mixer on the mixing process and its energy efficiency. 
Table 1. Foam concrete compositions of various densities. PC, Portland cement; FA, foaming agent.

\begin{tabular}{|c|c|c|c|c|c|c|c|c|c|}
\hline \multirow[t]{3}{*}{$\begin{array}{c}\text { Average } \\
\text { Density in } \\
\text { Dry State, } \\
\mathrm{kg} / \mathrm{m}^{3}\end{array}$} & \multicolumn{2}{|c|}{$\begin{array}{c}\text { Dry Mixture } \\
\text { Composition, } \\
\%\end{array}$} & \multicolumn{4}{|c|}{$\begin{array}{c}\text { Initial Components per } 1 \mathrm{~m}^{3}, \\
\mathrm{~kg}\end{array}$} & \multirow[t]{3}{*}{$\begin{array}{l}\text { Water-Solid } \\
\text { Ratio } \\
\text { (W/S) }\end{array}$} & \multirow[t]{3}{*}{$\begin{array}{c}\text { Density of Foam } \\
\text { at Filling } \\
\text { Products, } \\
\mathrm{kg} / \mathrm{m}^{3}\end{array}$} & \multirow[t]{3}{*}{$\begin{array}{c}\text { Compressive } \\
\text { Strength, } \\
\mathrm{MPa}\end{array}$} \\
\hline & \multirow[t]{2}{*}{ PC 500} & \multirow[t]{2}{*}{ Sand } & \multirow[t]{2}{*}{ PC 500} & \multirow[t]{2}{*}{ Sand } & \multicolumn{2}{|c|}{$\begin{array}{l}\text { The Amount of } \\
\text { FA, } \mathrm{L} / \mathrm{m}^{3}\end{array}$} & & & \\
\hline & & & & & FA1 & FA2 & & & \\
\hline 500 & 70 & 30 & 315 & 140 & 4.28 & - & 0.50 & 635 & 1.5 \\
\hline \multirow[t]{2}{*}{500} & 100 & - & 515 & - & 4.42 & - & 0.47 & 704 & 2.5 \\
\hline & 70 & 30 & 380 & 165 & 4.28 & - & 0.42 & 719 & 1.8 \\
\hline \multirow[t]{2}{*}{700} & 100 & - & 600 & - & 4.64 & - & 0.44 & 804 & 3.3 \\
\hline & 70 & 30 & 445 & 190 & 4.29 & - & 0.36 & 801 & 2.7 \\
\hline \multirow{2}{*}{900} & 50 & 50 & 420 & 420 & - & 3.18 & 0.26 & 1008 & 2.8 \\
\hline & 40 & 60 & 345 & 515 & - & 3.06 & 0.24 & 1003 & 2.6 \\
\hline \multirow[t]{2}{*}{1000} & 50 & 50 & 465 & 465 & - & 3.33 & 0.25 & 1070 & 5.8 \\
\hline & 40 & 60 & 380 & 565 & - & 3.08 & 0.22 & 1066 & 4.1 \\
\hline \multirow[t]{2}{*}{1100} & 50 & 50 & 515 & 515 & - & 3.37 & 0.23 & 1156 & 7.4 \\
\hline & 40 & 60 & 420 & 625 & - & 3.20 & 0.21 & 1185 & 5.7 \\
\hline \multirow[t]{2}{*}{1200} & 50 & 50 & 560 & 560 & - & 3.46 & 0.22 & 1287 & 9.3 \\
\hline & 40 & 60 & 460 & 685 & - & 3.49 & 0.20 & 1293 & 7.7 \\
\hline
\end{tabular}

The experiments were carried out using a flat-bottom apparatus. The apparatus with a diameter of $D=0.19 \mathrm{~m}$ was made of plexiglass and equipped with partitions along the axes, half immersed in the stirred medium. The width of the reflective partitions $b$ was $D / 10$, where $D$ is the diameter of the apparatus (Figure 2).

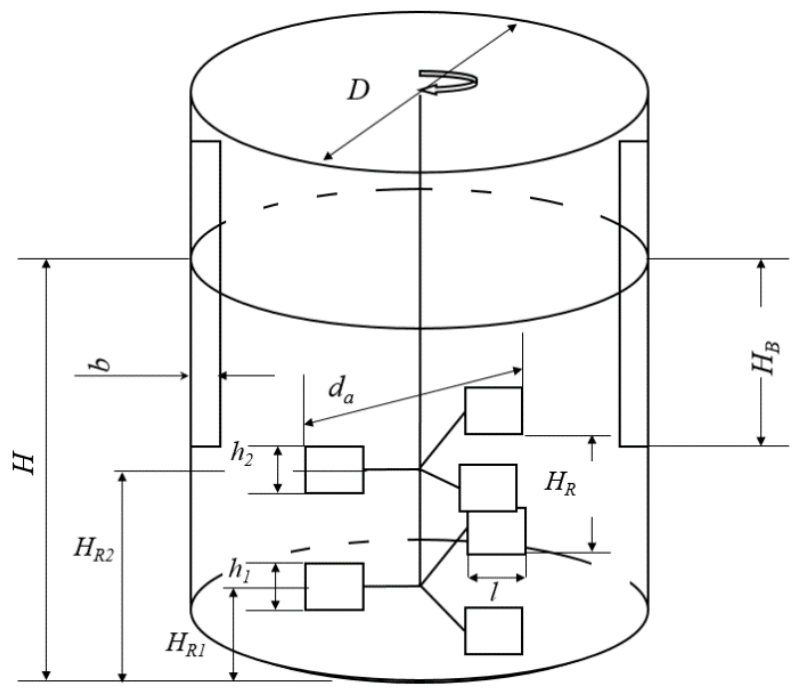

Figure 2. Scheme of apparatus with a two-row six-blade turbine mixer.

The height of the liquid level in the apparatus in relation to the diameter of the apparatus was $H / D=1.42$. As a mixing device $\left(d_{a}=0.42 D\right)$, two six-blade turbines were used with straight blades $\left(l=0.25 d_{a}\right), h_{1}=0.2 d_{a}$, as well as turbines with inclined blades (blades with an inclination angle equal to $45^{\circ}, h_{2}=0.25 d_{a}$ ). In the case of a two-row arrangement of the turbine on one shaft, four variants of the mutual combination of the types of agitator turbines were studied; the distance between them in all cases was $H_{R}=0.5 d_{a}$. Water (at $20^{\circ} \mathrm{C}$ ) was used as a dispersed medium; cement and sand with an average particle diameter of $d_{c e m}=312.3 \mu \mathrm{m}$ were applied as solid dispersible particles. 
The experiments were also carried out in vessels with a mixer, with diameters of $D=0.57 \mathrm{~m}$ and $D=1.0 \mathrm{~m}$; each vessel was equipped with four $0.9 \mathrm{D}$ wide partitions. Three types of agitators were used, namely, a standard disc turbine (SDT), blade turbine with downdraft (BTD), and vane turbine with upward flow (VTUF). The mixer rotation speed was varied in the range of $2-13.33 \mathrm{rad} / \mathrm{s}$. The experiment was carried out in a semiperiodic mode. The vessel was filled to a certain level $(H / D=1)$. The critical speed of mixing the suspension, $n_{c r}$, at which none of the particles remains at the bottom of the vessel for more than $2 \mathrm{~s}$, was defined.

To clarify the degree of influence of the configuration of the lower part of the turbulent mixer on the mixing efficiency and power consumption, studies were carried out on a mixer (see Figure 3) with a cylindrical part $D=520 \mathrm{~mm}$ and removable conical parts with inclination angles $\alpha_{\text {cone }}$ of $20^{\circ}, 30^{\circ}$, $40^{\circ}, 50^{\circ}, 60^{\circ}$, and $80^{\circ}$. The mixer was equipped with partition plates of $b=0.05 \mathrm{D}$ and a three-blade activator with straight blades; the activator rotation speed was $500 \mathrm{rpm}$. Note that the mixers with partitions provide high turbulence of the mixing process, which makes it possible to efficiently prepare a foam concrete mixture and uniformly distribute the components throughout the mixer.
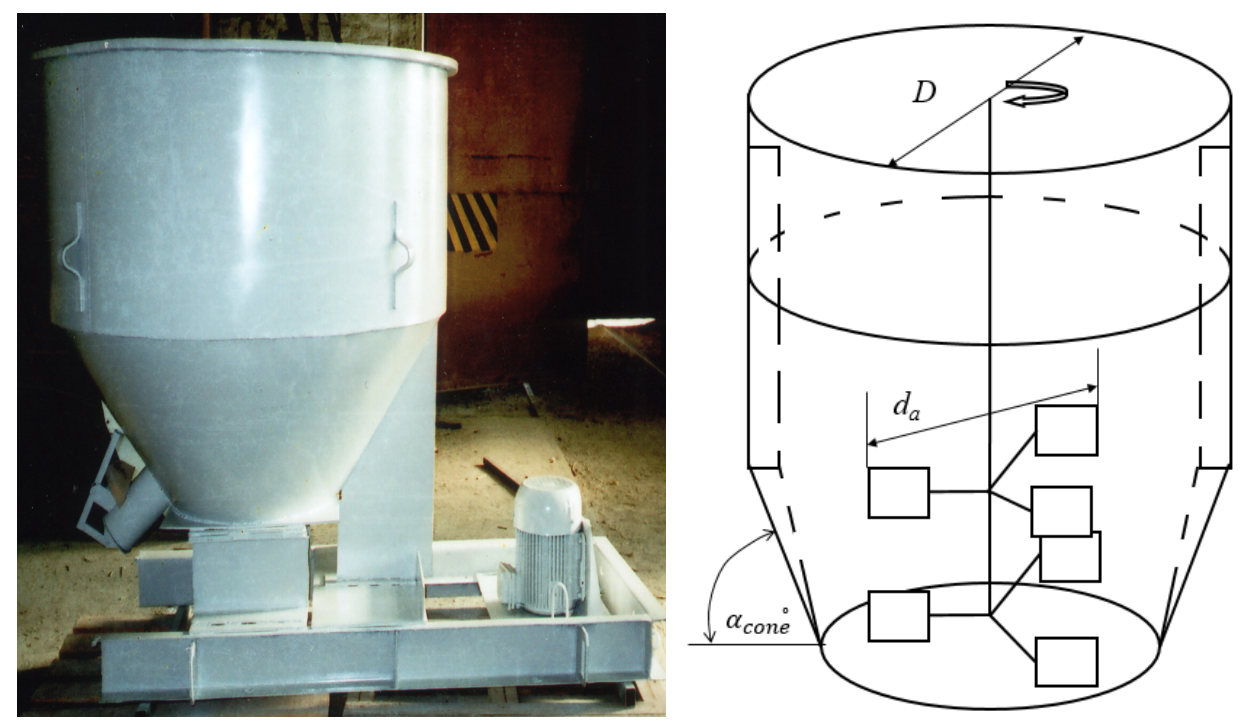

Figure 3. Cylindrical mixer with conical part.

The excited motion of the foam concrete mixture requires a continuous supply of energy. The dependence of the energy spent per unit of time (power) on the mixing conditions is usually expressed as follows [22]:

$$
N=K_{N} \rho n_{\text {act }}^{3} d_{a}^{5},
$$

where $N$ is the power $(\mathrm{W}), \rho$ is the medium density $\left(\mathrm{kg} / \mathrm{m}^{3}\right), n_{a c t}$ is the rotation speed of the activator $(\mathrm{rev} / \mathrm{s})$, and $d_{a}$ is the mixer (activator) diameter (m).

The coefficient $K_{N}$, called the power criterion [22], is generally variable. It depends on the type of activator, the size and design of the mixer, and the properties of the medium.

$$
K_{N}=3.87 Z_{m} \xi_{m} k_{N}
$$

where $Z_{m}$ is the number of stirrer blades (pcs), $\xi_{m}$ is the dimensionless resistance coefficient of the mixer, and $k_{N}$ is a dimensionless power consumption factor that relates the power to the characteristics of the circular flow of the foam concrete mixture in the mixer. In general, the coefficient $k_{N}$ can be calculated using the following expression [22]:

$$
k_{N}=\int_{\bar{r}_{b}}^{1}[\bar{r}-\bar{v}(r)]^{2} \bar{r} d \bar{r},
$$




$$
\begin{gathered}
\bar{r}_{b}=\frac{r_{b}}{r_{0}} ; \bar{r}=\frac{r}{r_{0}}, \\
\bar{v}(r)=\frac{v(r)}{\omega_{0} r_{0}},
\end{gathered}
$$

where $r_{b}$ is the radius of the inner edges of blades $(\mathrm{m}), \bar{r}_{b}$ is the relative radius of the inner edges of blades, $\bar{r}$ is the relative average distance from the vertical axis of vehicle, $\bar{v}(r)$ is the relative average peripheral velocity of stirred medium at a distance $r$ from the vertical axis of the apparatus, $\omega_{0}$ is the angular velocity of the mixer ( $\mathrm{rad} / \mathrm{s}), v(r)$ is the circumferential velocity of the liquid at a distance $r$ $(\mathrm{m} / \mathrm{s})$, and $r_{0}$ is the stirrer radius $\left(r_{0}=d_{\mathrm{a}} / 2\right)(\mathrm{m})$.

Experimentally, the refinement of the coefficient $k_{N}$ can be described as

$$
k_{N}=\frac{N_{0}}{N_{\text {consum }}},
$$

where $N_{0}$ is the power consumption of the mixer without loading the mixture $(\mathrm{W})$, and $N_{\text {consum }}$ is the power consumed by the mixer for mixing the mixture $(\mathrm{W})$.

The power $N(\mathrm{~W})$ consumed for mixing can be expressed as

$$
N=\omega_{0} M_{t}
$$

where $\omega_{0}$ is the angular velocity of the mixer $(\mathrm{rad} / \mathrm{s})$, and $M_{t}$ is the torque applied to the blades $(\mathrm{N} \cdot \mathrm{m})$.

The relationship between the value of $M_{t}(\mathrm{~N} \cdot \mathrm{m})$ (and the distribution of the peripheral speed is described by the following relationship [22]:

$$
M_{t}=Z_{m} \xi_{m} \rho \frac{\omega_{0}^{2} r_{0}^{5}}{2} \int_{\bar{r}_{b}}^{1}[\bar{r}-\bar{v}(r)]^{2} \bar{r} d \bar{r}
$$

where $Z_{m}$ is the number of agitator blades, varying from $2-8$, and $\xi_{m}=0.88$ is a dimensionless blade resistance coefficient.

Obviously, the occurrence of torque is caused by the presence of a speed difference between the blades of the mixer and the foam concrete mixture. The torque is equal to the moment of the hydraulic resistance forces of the blades for a flux around them.

Taking into account that $\omega_{0}=2 \pi n_{\text {act }}$, we have

$$
N=3.87 Z_{m} \xi_{m} \rho n_{a c t}^{3} d_{a}^{5} \int_{\bar{r}_{b}}^{1}[\bar{r}-\bar{v}(r)]^{2} \bar{r} d \bar{r}
$$

By using the rotational speed of the activator $n_{\text {actrpm }}$ in rpm, $n_{\text {act }}=n_{\text {actrpm }} / 60$, and we have

$$
N=1.79 \times 10^{-5} Z_{m} \xi_{m} \rho n_{a c t r p m}^{3} d_{a}^{5} \int_{\bar{r}_{b}}^{1}[\bar{r}-\bar{v}(r)]^{2} \bar{r} d \bar{r},
$$

where the power $N$ represented in Equations (9) and (10) has a dimension of $W$ with the corresponding dimension of the parameters described earlier.

In Equations (7)-(10), all design features of the mixers are taken into account by the values of the resistance coefficient $\xi_{m}$, which is determined from the literature [22]. The value of $\xi_{m}$ for mixers of a given geometry in a turbulent regime remains constant, and the task is to determine the coefficient $k_{N}$, which relates the power to the characteristics of the circular flow of the foam concrete mixture in the mixer.

\section{Results and Discussion}

We divided our research into seven stages. 
At the first, second, fourth, and fifth stages, a cylindrical mixer was used (Figure 2), while at the third, sixth, and seventh stages, a cylindrical mixer with a conical part was used (Figure 3).

Mixer configurations used were as follows:

Stage 1: $\mathrm{D}=0.57 \mathrm{~m}, \mathrm{H}=1.15 \mathrm{~m}$, with a downdraft turbine blade without baffles;

Stage 2: $\mathrm{D}=0.57 \mathrm{~m}, \mathrm{H}=1.15 \mathrm{~m}$, with up-flow turbine impeller with baffles;

Stage 3: $\mathrm{D}=0.52 \mathrm{~m}, \mathrm{H}=0.78 \mathrm{~m}, \mathrm{~V}=250 \mathrm{~L}$, with baffles and replaceable tapered parts with different inclination angles;

Stage 4: $\mathrm{D}=0.19 \mathrm{~m}, \mathrm{H}=0.42 \mathrm{~m}$, with a downdraft turbine impeller with baffles and a variable number of blades;

Stage 5: $\mathrm{D}=0.19 \mathrm{~m}, \mathrm{D}=0.57 \mathrm{~m}, \mathrm{D}=1.0 \mathrm{~m}$, with different types of mixers with baffles;

Stage 6: $\mathrm{D}=0.52 \mathrm{~m}, \mathrm{H}=0.78 \mathrm{~m} \mathrm{~V}=250 \mathrm{~L}$, three-blade activator with replaceable blades;

Stage 7: $\mathrm{D}=0.52 \mathrm{~m}, \mathrm{H}=0.78 \mathrm{~m}, \mathrm{~V}=250 \mathrm{~L}$, with three plain blades with variable blade pitch.

\subsection{Effect of the Ratio of Mixture Height to Mixer Diameter on the Quality of Mixture and Power Consumption} (Stage 1)

In practice, the height of the foam concrete mixture in the mixer vessel $\left(H_{m i x}\right)$ (for blade mixers) is taken equal to the vessel diameter $(D)$, that is, $H_{m i x}=D$. However, in the case of designing a mixer having another height of mixture in the vessel, the following question arises: Could this circumstance influence a homogeneity of the mixture density and a consumed power for mixing?

Obviously, the parameter $H_{\text {mix }} / D$ affects the density of a foam concrete mixture. Figure 4 shows the dependence of the homogeneity coefficient of the foam concrete mixture, as a function of the dependence of the density parameter $K_{c h m}=\bar{\rho}_{\min } / \bar{\rho}_{\max }$ on the ratio $H_{m i x} / D$. The experiment was carried out on a cylindrical mixer with a conical bottom. The density of the foam concrete mixture in the mixer was measured using the probes of mixture after its preparation. This operation was carried out through the discharge valves installed on the mixer body along the entire height with a step of $300 \mathrm{~mm}$.

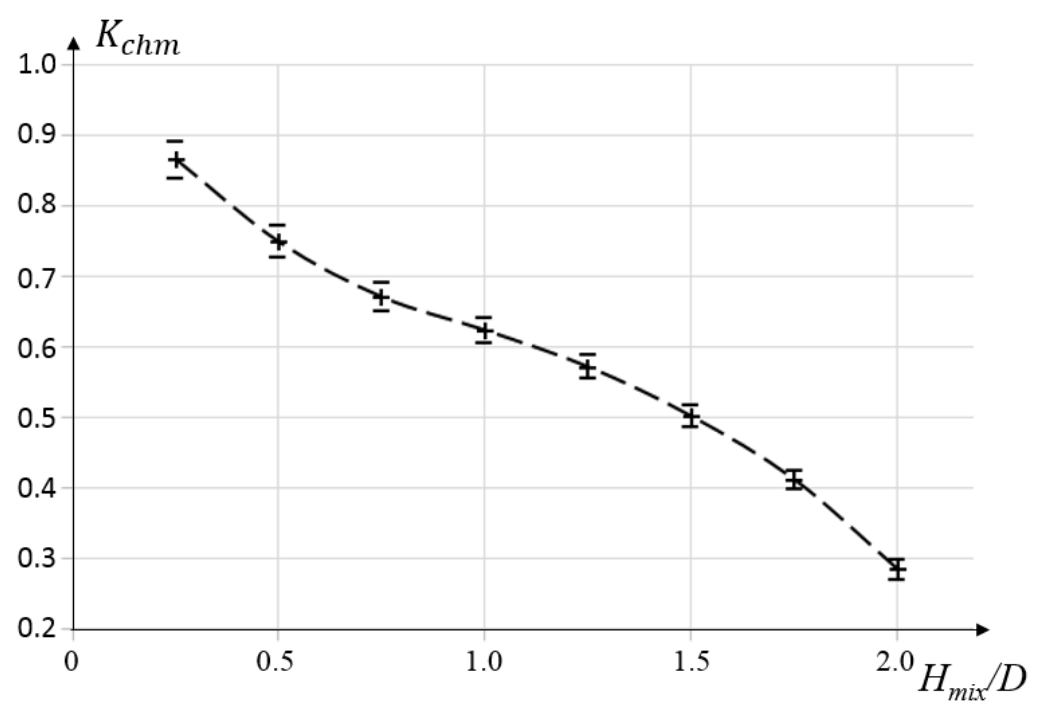

Figure 4. Homogeneity of foam concrete mixture $K_{c h m}$ vs. ratio $H_{m i x} / D$ (experimental data).

Figure 4 shows that the homogeneity of the mixed mixture steadily decreased with an increase in the height of the mixture loading. This is explained by the fact that the circulation flow rate $q$ decreased with an increase in the height of mixture, the movement of the mixture changed from a turbulent regime to a laminar one, and the turbulent transfer of the mixture particles between the central zone and the peripheral zone decreased. Then, an increase in the mixture height led to an increase in the 
power consumption factor $k_{N I}$ (subscript $I$ defines the corresponding stage of the study), which was calculated at the $I$-th stage as $k_{N I}=N_{0} / N_{\text {consum }}$.

The dependence of $k_{N 1}$ on the parameter $H_{m i x} / D$ indicates that an increase in the filling height of the mixture with a constant diameter led to an increase in the power consumption of the mixer (Figure 5).

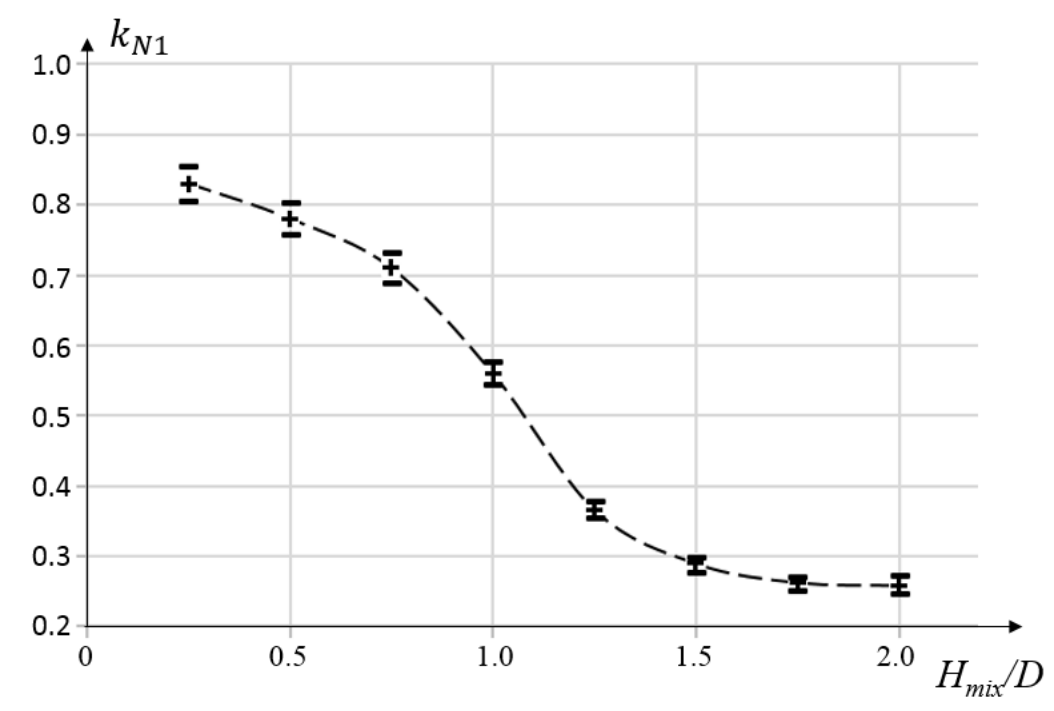

Figure 5. Influence of the ratio $H_{m i x} / D$ on the power $k_{N 1}$ spent on mixing (experimental data).

The coupled analysis of the effects of $H_{m i x} / D$ on the homogeneity coefficient of the mixture $K_{c h m}$ and on the power factor $k_{N 1}$ shows that, with an increase in the loading height of the foam concrete mixture at the ratio $H_{m i x} / D$ from 1 to 2, there was a sharp decrease in the homogeneity of the mixture. This indicates a decrease in the mixing efficiency in the upper zone of the mixture. At the same time, starting from the value of the ratio $H_{m i x} / D=1.5$, some stabilization of $k_{N 1}$ was observed. This indicates that a further increase in the mixture height led to a decrease in the efficiency of mixing and aeration of the upper layers of the mixture. Thus, the parameter $H_{\text {mix }} / D$ in the range of $0.75-1$ is optimal for a foam concrete mixture.

\subsection{Effect of Reflective Partitions on the Quality of Foam Concrete Mixture and Power Consumption (Stage 2)}

The effect of the number of partitions $(J)$ and the ratio of the partition width to the mixer diameter $(b / D)$ on the power consumed for mixing was discussed in [22,23]. In [24], it was shown that the power consumed for mixing increases with an increase in the number and width of reflective partitions. In [22], it was shown that, with an increase in the width and number of partitions, the maximum power consumed for mixing is achieved. Moreover, these authors showed that a slight inclination of the partitions or their distance from the vessel walls did not significantly affect the power spent on mixing and mixture quality.

Determination of the effect of the normalized width and the number of partitions on the power factor of the mixer drive $k_{N 2}$ and the homogeneity coefficient $K_{c h m}$ of foam concrete mixture was carried out in a cylindrical mixer with a conical part and three-blade activator by mixing a foam concrete mixture of constant composition. The constant rotation speed of the activator was equal to $500 \mathrm{rpm}$. The obtained experimental results are shown in Figures 6 and 7. 


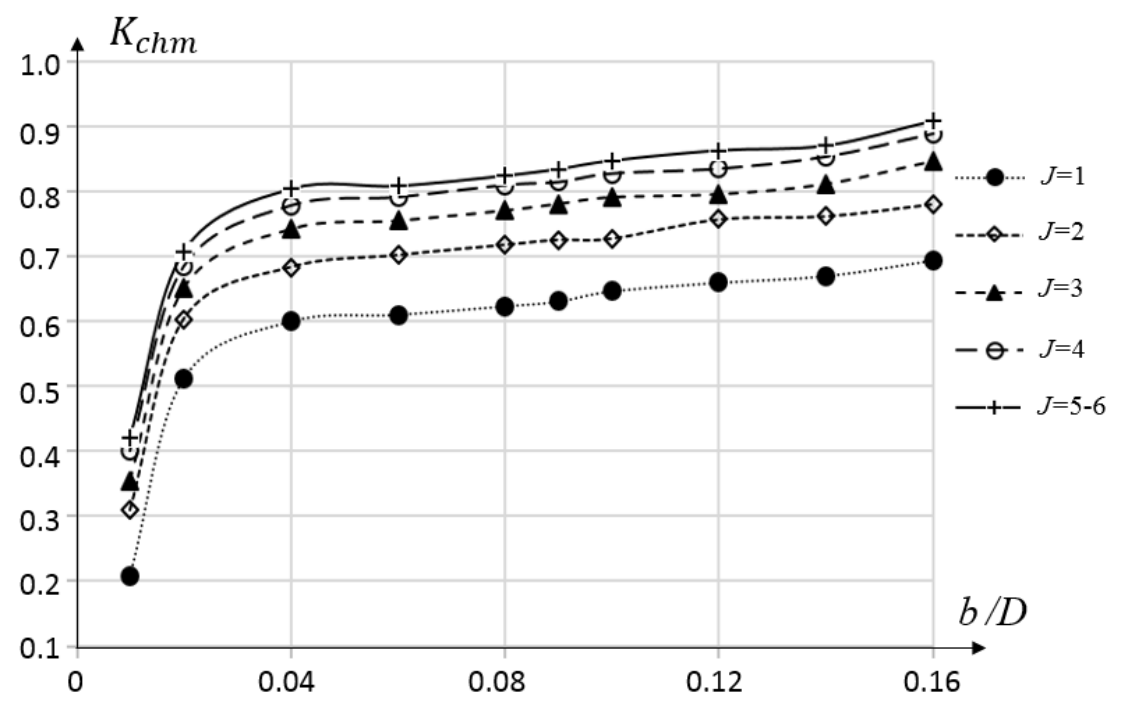

Figure 6. Uniformity coefficient $K_{c h m}$ of foam concrete mixture vs. normalized width $b / D$ and number $J$ of reflective partitions (experimental data).

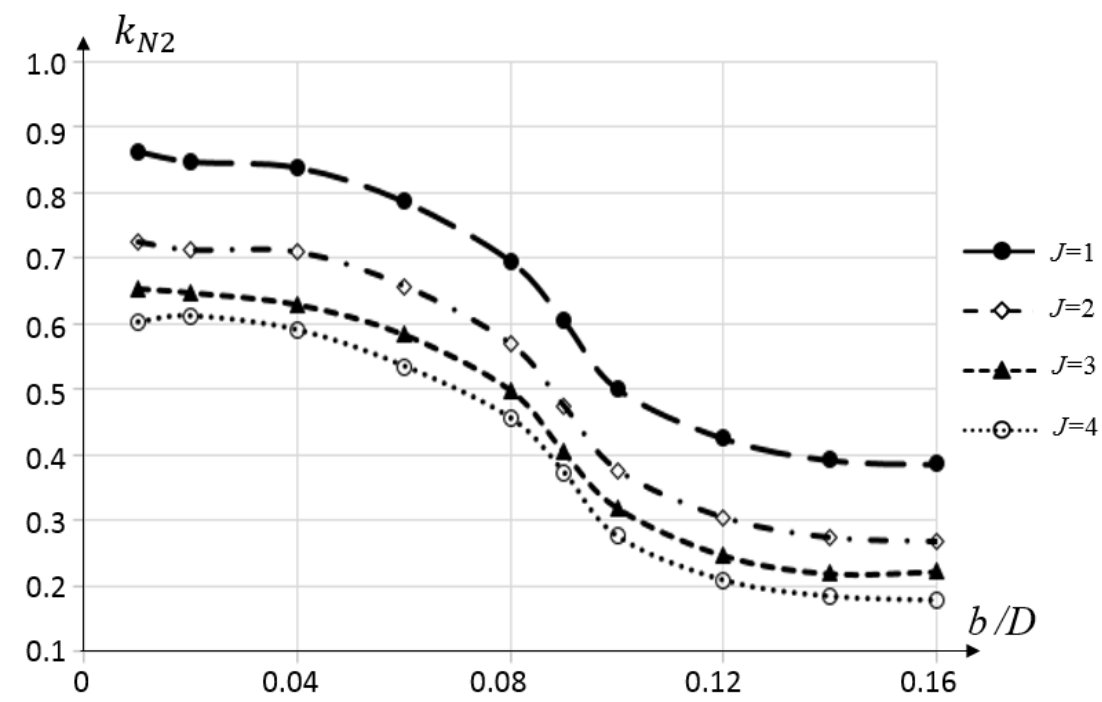

Figure 7. Influence of parameter $b / D$ on the power factor of mixer drive $k_{N 2}$ (experimental data).

Analysis of the data shows that, with an increase in the width of the reflective partitions, the power consumed for mixing increased. The homogeneity coefficient of the mixture sharply increased to the values of $b / D=0.04-0.06$, while a further increase in the width of the reflective partitions insignificantly affected the increase in the homogeneity of the mixture. According to the data of Figure 7, it can be concluded that, in the range of $b / D=0.04-0.06$ with lower energy consumption, it is possible to obtain a high-qualitative (homogeneous throughout the volume) foam concrete mixture.

\subsection{Effect of the Shape of the Conical Part of Mixer on the Homogeneity of Foam Concrete Mixture and Power Consumption (Stage 3)}

Until now, there have been no systematic studies that could give an answer to the question of how the shape of mixer affects power consumption and mixing efficiency [25-29].

To determine the influence of the of the lower part of the turbulent mixer on mixing efficiency and power consumption, the studies were carried out on a mixer with a cylindrical part and removable conical parts (Figure 3). Power and density were measured similarly to the first stage of the study. The experimental results are shown in Figures 8 and 9. 


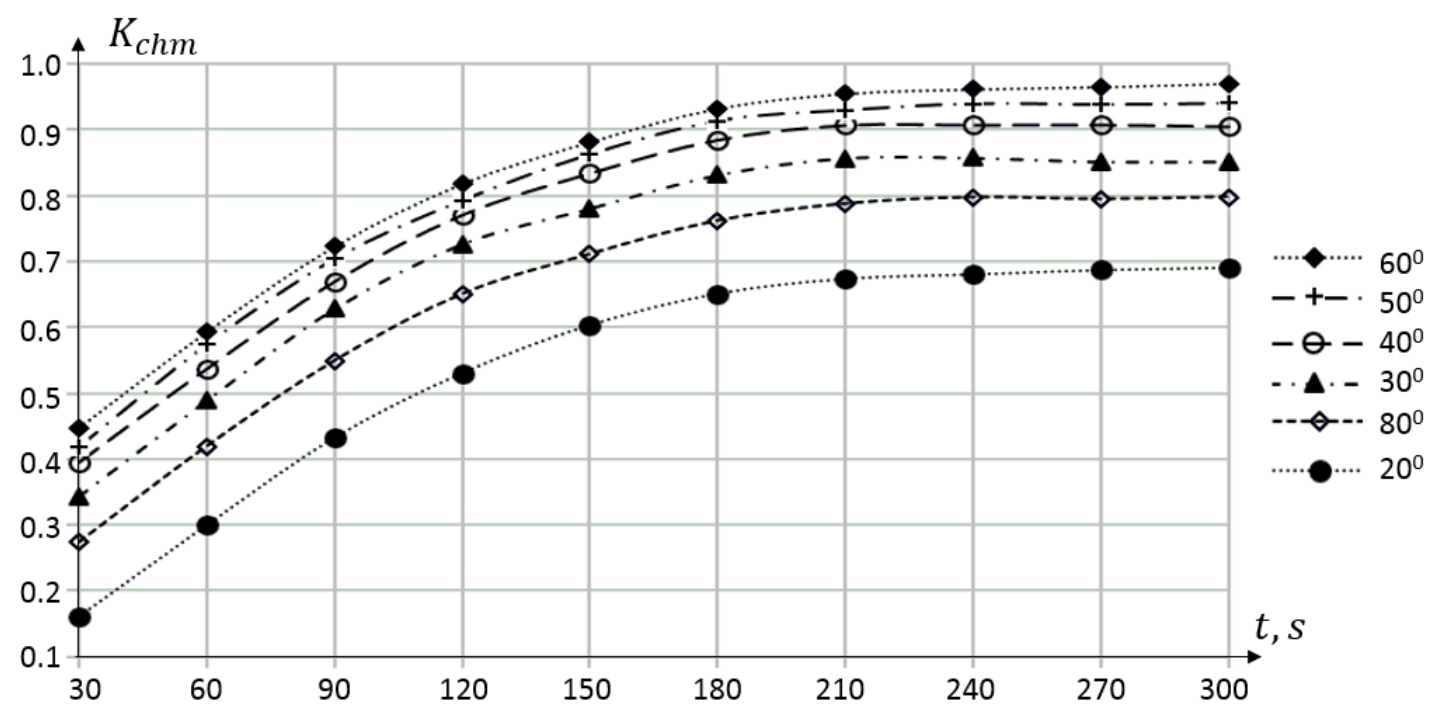

Figure 8. Homogeneity coefficient of foam concrete mixture vs. mixing time $t$ at different tilt angles of the conical part of turbulent mixer (experimental data).

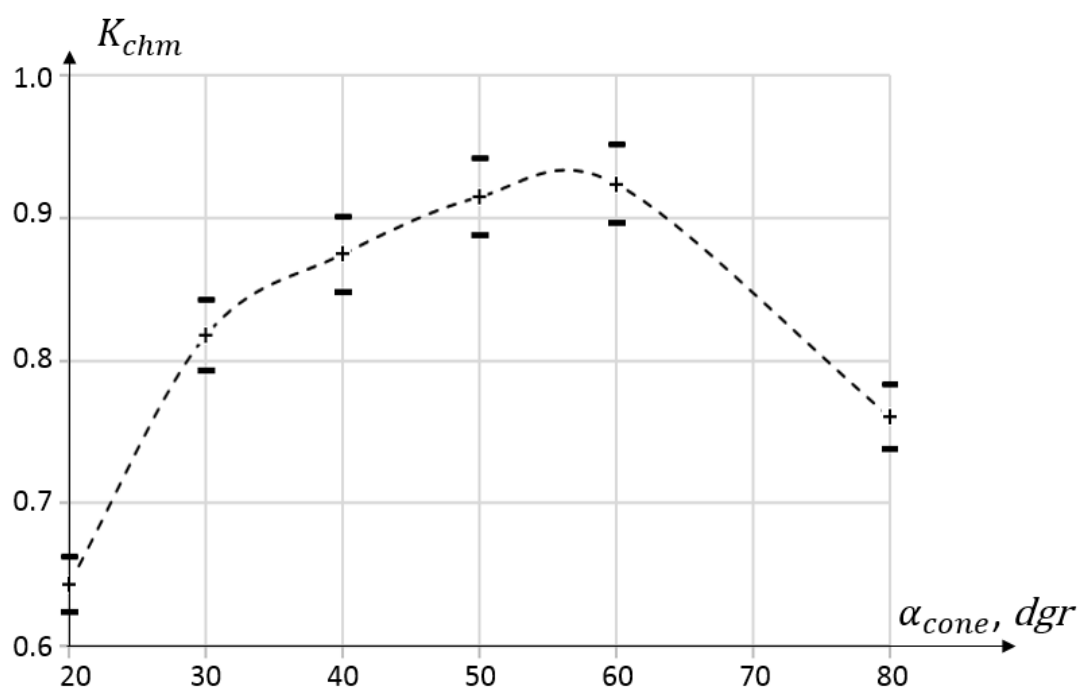

Figure 9. Homogeneity coefficient of foam concrete mixture vs. inclination angle $\alpha_{\text {cone }}$ of the conical part of turbulent mixer at fixed mixing time $t=180 \mathrm{~s}$ (experimental data).

Analysis of the results shows that an increase in the preparation time of foam concrete mixture up to $5 \mathrm{~min}$ led to an increase in its homogeneity, regardless of the angle of inclination $\alpha_{\text {cone }}$ of the conical part of mixer (Figure 8). However, for a fixed mixing time (Figure 9, at $t=180 \mathrm{~s}$ ), a change in the angle of inclination of the conical part $\alpha_{\text {cone }}$ from $20^{\circ}$ to $60^{\circ}$ led to an increase in the quality of mixture. With an increase in the angle of inclination $\alpha_{\text {cone }}$ over $60^{\circ}$, a decrease in the homogeneity coefficient was observed, explained by a decrease in the axial component of turbulent mixing velocity.

The analysis of the power consumption for the preparation of foam concrete mixture shows that an increase in the angle $\alpha_{\text {cone }}$ from $20^{\circ}$ to almost $60^{\circ}$ led to an increase in the values of $k_{N 3}$ (Figure 10). A further increase in the angle $\alpha_{\text {cone }}$ of inclination of the conical part led to a sharp increase in power consumption. 


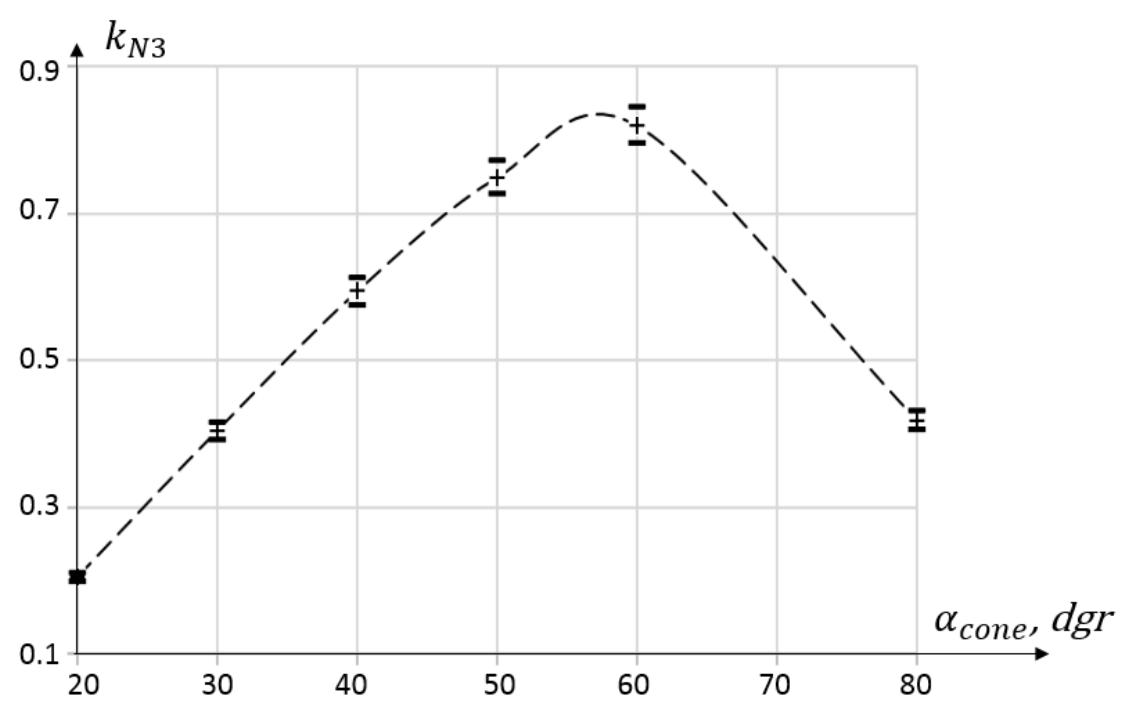

Figure 10. Influence of the inclination angle of conical part $\alpha_{\text {cone }}$ on the power consumption factor of mixer $k_{N 3}$ (experimental data).

3.4. Effect of the Number of Activator Blades on Mixture Homogeneity and Power Consumed for Mixing the Foam Concrete Mixture (Stage 4)

At this stage, the influence of the number of activator blades on the homogeneity of the foam concrete mixture was investigated. For this, the following actions were performed: the samples were made from the working version of the mixture composition in series of $n_{s m p}=10$ pieces for each version of the blades. The number of variations in the mixture composition was nine pieces. The recipe was selected for testing with an average density of mixtures from 400 to $1200 \mathrm{~kg} / \mathrm{m}^{3}$ with a step of $100 \mathrm{~kg} / \mathrm{m}^{3}$. The experimental composition of the mixtures is shown in Table 1. Mixing took place for $360 \mathrm{~s}$. After the mixing, a finished mixture was taken from the mixer and poured into test forms in the form of a cube. The size of the test pieces was $100 \times 100 \times 100 \mathrm{~mm}^{3}$. The density of foam concrete was determined by testing samples dried to constant weight under normal conditions. The sample density values were obtained for each batch test for each mixture and paddle option. For each sample from the working version of the mixture composition, three extreme terms were selected to estimate the maximum and minimum density values. The calculation of the averaged maximum $\bar{\rho}_{\max }$ and averaged minimum $\bar{\rho}_{\text {min }}$ values of the density of the finished samples took place. The mean density value was estimated as $\bar{\rho}=\sum \rho_{i} / n_{s m p}$. Then, the coefficient of homogeneity of the foam concrete mixture $K_{c h m}$ was calculated. The test results and estimates of variations in the homogeneity coefficient of the foam concrete mixture $K_{\text {chm }}$ are shown in Table 2.

Table 2. Dependence of the homogeneity coefficient of foam concrete mixture on the change in the number of activator blades and rotation speed.

\begin{tabular}{ccccc}
\hline \multirow{2}{*}{ No. Number of Blades, pcs } & \multicolumn{3}{c}{ Coefficient of Homogeneity of Foam Concrete Mixture $\boldsymbol{K}_{\text {chm }}=\bar{\rho}_{\text {min }} / \boldsymbol{\rho}_{\text {max }}$} \\
\cline { 3 - 5 } & & \multicolumn{3}{c}{ Activator Rotation Speed, rpm } \\
\cline { 3 - 5 } & 2 & $0.52 \pm 0.03$ & $\mathbf{5 0 0}$ & $\mathbf{8 0 0}$ \\
\hline 1 & 3 & $0.63 \pm 0.03$ & $0.59 \pm 0.03$ & $0.63 \pm 0.03$ \\
2 & 4 & $0.64 \pm 0.03$ & $0.96 \pm 0.04$ & $0.96 \pm 0.04$ \\
3 & 5 & $0.65 \pm 0.03$ & $0.96 \pm 0.04$ & $0.96 \pm 0.04$ \\
4 & 6 & $0.65 \pm 0.03$ & $0.96 \pm 0.04$ & $0.96 \pm 0.04$ \\
5 & 7 & $0.66 \pm 0.03$ & $0.96 \pm 0.04$ & $0.96 \pm 0.04$ \\
6 & 8 & $0.65 \pm 0.03$ & $0.95 \pm 0.04$ & $0.92 \pm 0.04$ \\
7 & & &
\end{tabular}


The initial analysis of the results showed that the homogeneity coefficient of the foam concrete mixture $K_{c h m}$ at a rotational speed of $200 \mathrm{rpm}$ did not exceed 0.7 for all variants of the mixture compositions. The average spread of values $K_{c h m}$ did not exceed $5 \%$.

This analysis showed that the effect of the number of blades of the activator $\left(Z_{m}\right)$ on the homogeneity degree of mixed foam concrete was insignificant if $Z_{m} \geq 3$ pcs. This was shown by our measurements of the density of foam concrete mixture in mixer after its preparation.

Analyzing these results, we found that increasing the number of blades from two to three significantly increased the homogeneity of the mixture being prepared. A further increase in the number of blades did not lead to a significant improvement in the quality of foam concrete mixture.

Thus, both from the viewpoint of the homogeneity of the prepared foam concrete mixture and from the viewpoint of the design of the activator, the optimal number of blades was $Z_{m}=3$ pcs.

As a result of our studies, it turned out that, with an increase in the number of activator blades, the power spent on mixing the mixture increased. Figure 11 shows the dependence of the power consumption factor $k_{N 4}$ on the number of revolutions of the activator $n$ and the number of blades $Z_{m}$ at activator diameter $d_{a}=$ const for an activator with straight blades. The studies were carried out for $Z_{m}$ $=2-8$ blades.

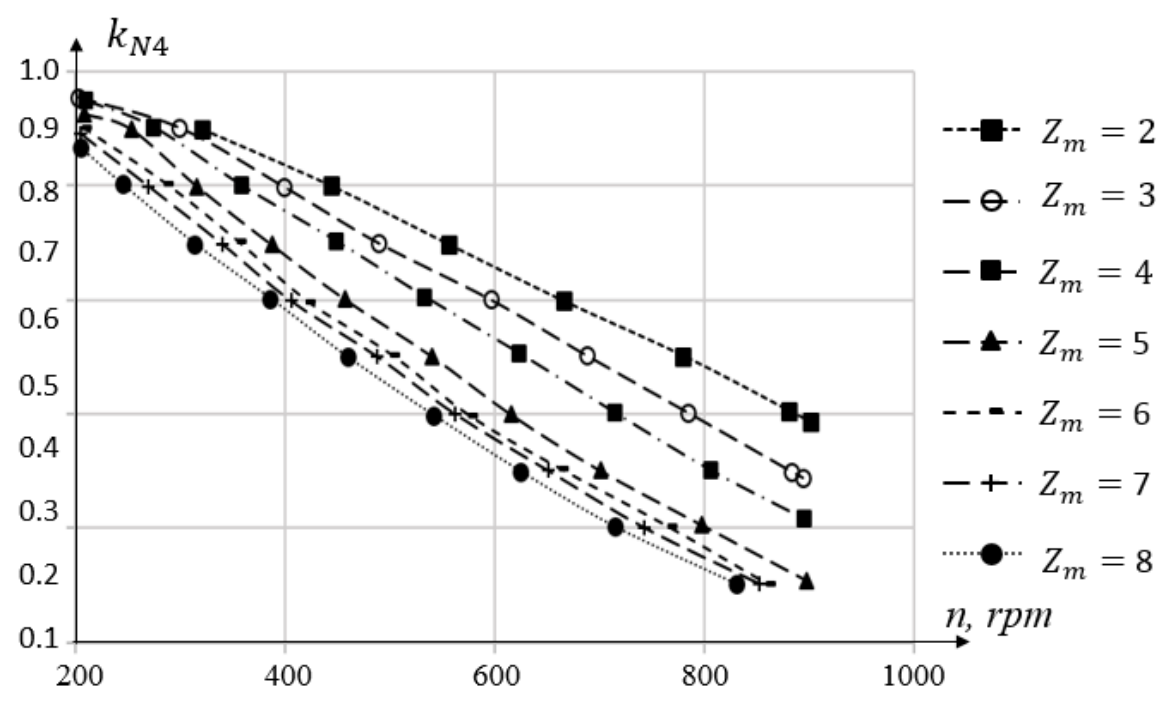

Figure 11. Influence of the number of blades $\left(Z_{m}\right)$ on the power consumed by activator $\left(k_{N 4}\right)$ with straight blades at different speeds of rotation $(n)$ (experimental data).

The obtained dependences show that an increase in the number of blades of the activator and the speed of its rotation led to an increase in the power spent on mixing. Thus, an increase in the number of blades from three to six led to an increase in power by about $30 \%$. The final decision of the number of activator blades should be made taking into account the data in Figure 11 and the economic efficiency of the adopted design decisions.

\subsection{Effect of the Ratio of Mixer Diameter to Activator Diameter on the Homogeneity of Foam Concrete Mixture and Power Consumption (Stage 5)}

Changing the ratio of mixer diameter to activator diameter in the range $D / d_{a}=1-7$ influences the power spent on mixing insignificantly if there are no reflective partitions in the mixer design. If there are reflective partitions in the mixer, this effect is enhanced. According to the results of previous studies, the optimal value of the parameter $J b / D=0.25$ was selected. In this case, we carried out experimental studies of the influence of the parameter $D / d_{a}$ on the power consumed by the mixer. The number of reflective partitions in the mixer was $J=4$, with a width of $b=0.06 D$. The research results are shown in Figure 12. 


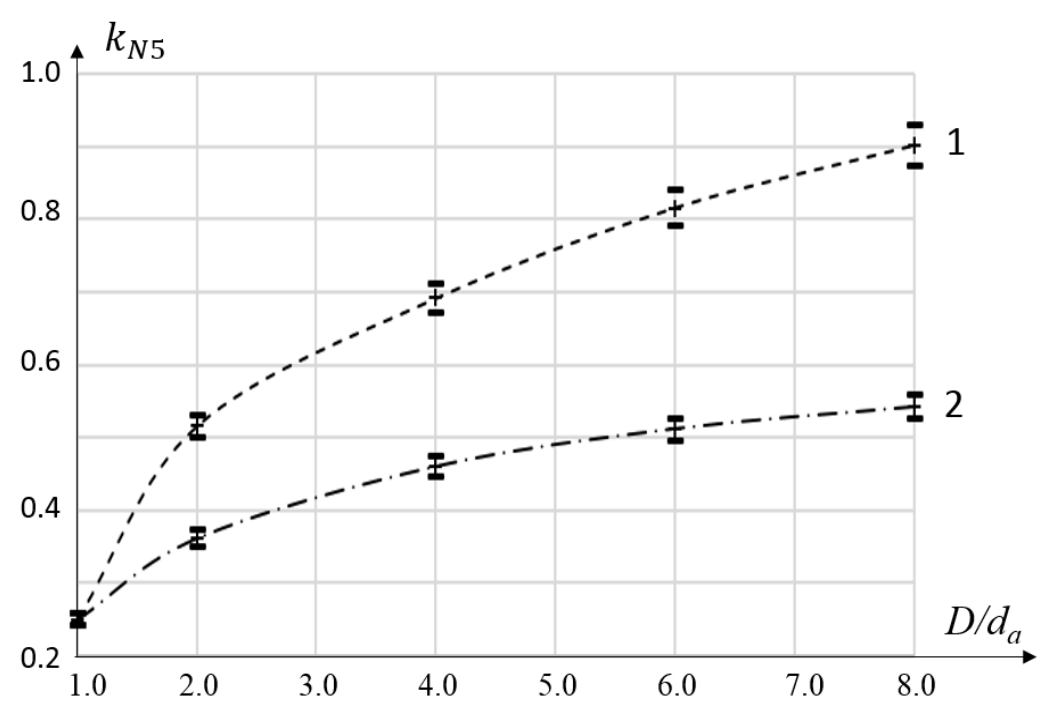

Figure 12. Influence of the ratio $D / d_{a}$ on the power factor $k_{N 5}: 1-$ mixer design without partition plates; 2 -mixer design with reflective partitions $(J=4)$ (experimental data).

By analyzing the results, it can be seen that an increase in the parameter $D / d_{a}$ (a decrease in the diameter of the activator) reduced the power consumption. To assess the degree of influence of $D / d_{a}$ on the homogeneity of the foam concrete mixture, we carried out an experiment on a laboratory mixer of cylindrical shape $(D=150 \mathrm{~mm})$ with a conical part $\left(\alpha_{\text {cone }}=60^{\circ}\right)$. Reflective partitions with a width of $b$ $=0.06 \mathrm{D}$ were installed in the mixer. The experimental results are shown in Figure 13.

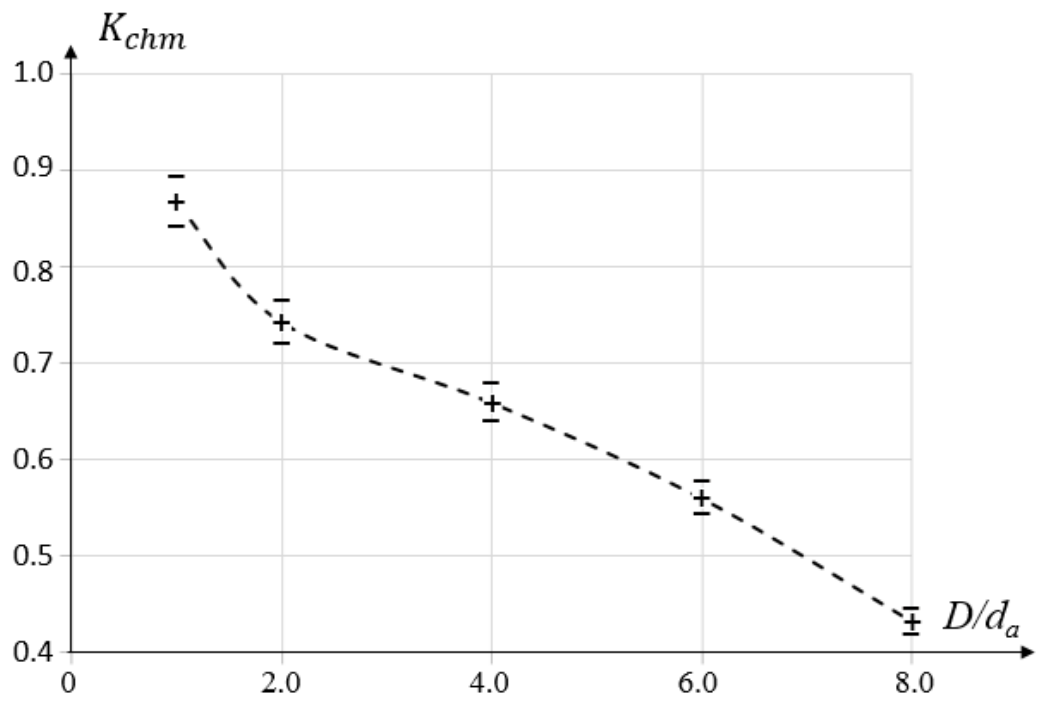

Figure 13. Mixture homogeneity coefficient $K_{c h m}$ vs. parameter $D / d_{a}$ (experimental data).

Figure 13 shows that an increase in the ratio $D / d_{a}$ led to a decrease in the homogeneity of the foam concrete mixture. The results of experiments assessing the degree of influence of the parameter $D / d_{a}$ showed that the choice of the ratio of the mixer diameter to the activator diameter should be carried out taking into account the power consumption $k_{N 5}$ and the homogeneity of the prepared mixture $K_{c h m}$. The research results demonstrate that the optimal value of the ratio $D / d_{a}$ ranged from 4-6. 
3.6. Effect of the Blade Sizes on the Mixing Quality of Mixture and the Power Consumption of Mixer Drive (Stage 6)

The study of the influence of the geometric parameters of mixer on the mixture quality and the power consumption revealed the need to take into account the geometric parameters of the activator. Such parameters are the sizes of blades (length $a_{b l}$ and width $b_{b l}$ ), measured from a projection onto a plane passing through the axis of the mixer shaft and the middle of a blade (Figure 14).

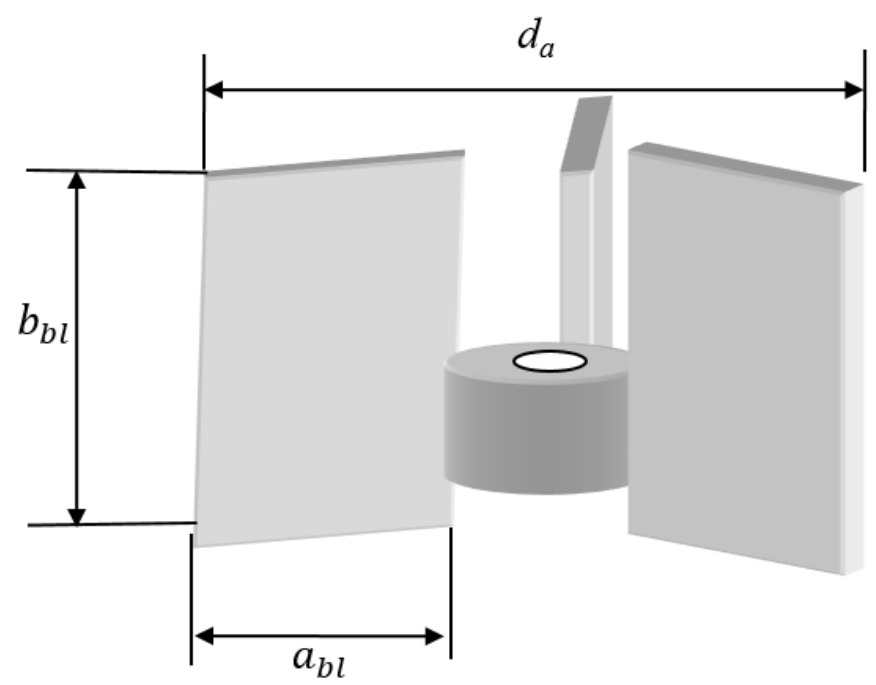

Figure 14. Geometric parameters of blades.

At the next stage of the experiment, a cylindrical mixer with a conical part and a three-blade activator with removable blades were used. The blades were chosen with $a_{b l} / b_{b l}$ ratio ranging from 0.2 to 0.6. The constant speed of rotation of the activator was taken equal to $500 \mathrm{rpm}$. A foam concrete mixture of the following composition per $1 \mathrm{~m}^{3}$ was used as a stirred medium: cement-400 kg; sand-200 kg.; water-480 L; foaming agent-1 L [27-29].

The dependence of the homogeneity coefficient of the mixture $K_{c h m}$ on the parameter $a_{b l} / b_{b l}$ is shown in Figure 15, and a graph of power factor $k_{N 6}$ versus parameter $a_{b l} / b_{b l}$ is shown in Figure 16.

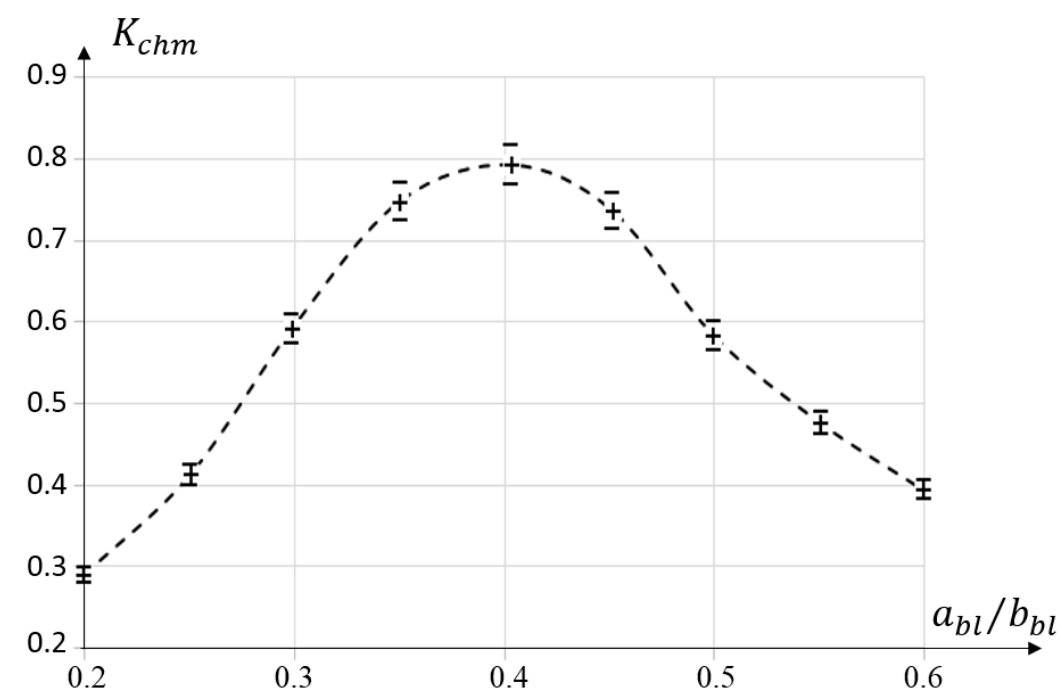

Figure 15. Homogeneity coefficient of mixture $K_{c h m}$ vs. parameter $a_{b l} / b_{b l}$ (experimental data). 


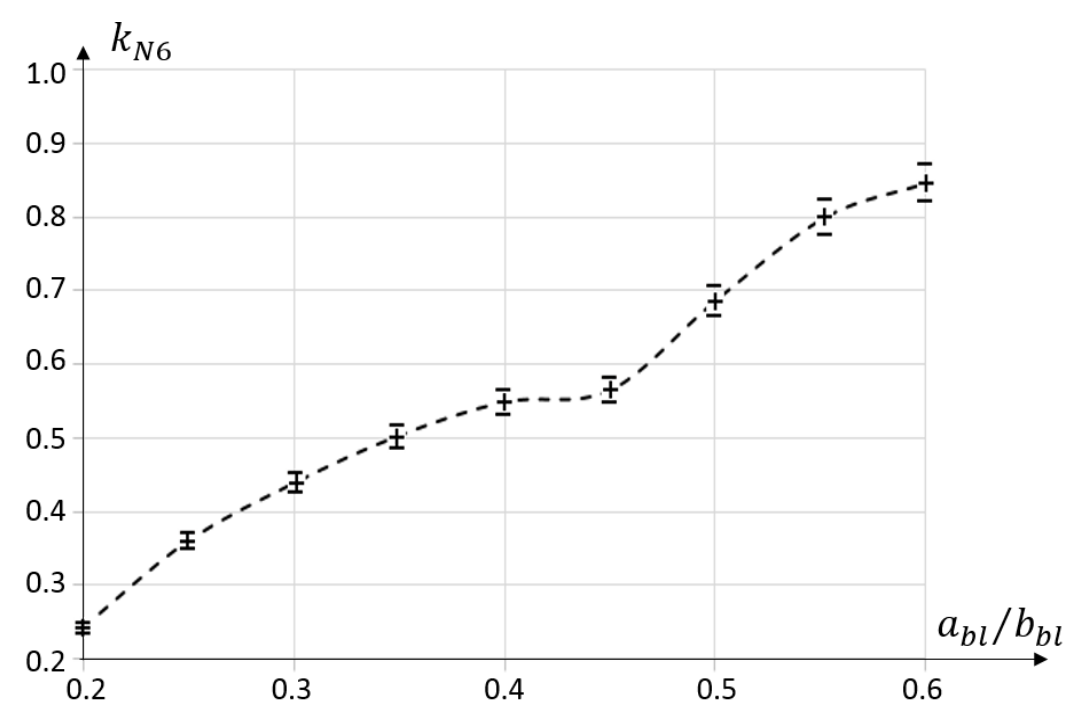

Figure 16. Influence of the parameter $a_{b l} / b_{b l}$ on the power factor $k_{N 6}$ (experimental data).

The dependence shows that the maximum value of $K_{c h m}$ was achieved at $a_{b l} / b_{b l}$ from 0.35 to 0.45 . Therefore, in the specified range of $a_{b l} / b_{b l}$, the maximum intensity of mixing of the mixture was achieved. The mixture moved from laminar to turbulent regime. The rate of mass transfer of the foam concrete mixture in the axial direction increased. Moreover, an increase in the parameter $a_{b l} / b_{b l}$ led to an increase in power consumption. In the $a_{b l} / b_{b l}$ range of $0.35-0.45$, some stabilization of $k_{N 6}$ was observed.

Therefore, the optimal value of the $a_{b l} / b_{b l}$ parameter, which ensured the maximum homogeneity of the foam concrete mixture at optimal values of $k_{N 6}$, was in the range of $a_{b l} / b_{b l}$ ratios from $0.35-0.45$.

3.7. Effect of the Inclination of Activator Blades on the Homogeneity of Foam Concrete Mixture and the Power Consumption of Mixer Drive (Stage 7)

Earlier, this effect was studied in $[25,27]$. To assess the influence of the inclination of activator blades relative to the plane perpendicular to the rotation axis of activator on the homogeneity of foam concrete mixture and the power spent on mixing the foam concrete mixture, a number of experiments were carried out. In the experiments, a cylindrical mixer with a conical part $V=250 \mathrm{~L}$ was used, and the rotation speed of the activator was $500 \mathrm{rpm}$. Blade activators with three flat blades were used. On each of the activators, the blades were installed radially and inclined at an angle $\alpha_{l}=20^{\circ}, 30^{\circ}, 45^{\circ}$, $50^{\circ}$, and $60^{\circ}$ relative to the plane perpendicular to the axis of rotation of the mixer (for straight blades, $\left.\alpha_{l}=0^{\circ}\right)$.

The study of the influence of the inclination angle of blades $\alpha_{l}$ on the uniformity coefficient $K_{c h m}$ showed that the homogeneity of foam concrete mixture decreased with an increase in the inclination angle of blades (see Figure 17). This was due to a decrease in the rotation speed of the mixture in accordance with the intensity of mixing. With an increase in the inclination angle of blades $\alpha_{l}$, the power $k_{N 7}$ spent on mixing decreased (Figure 18). 


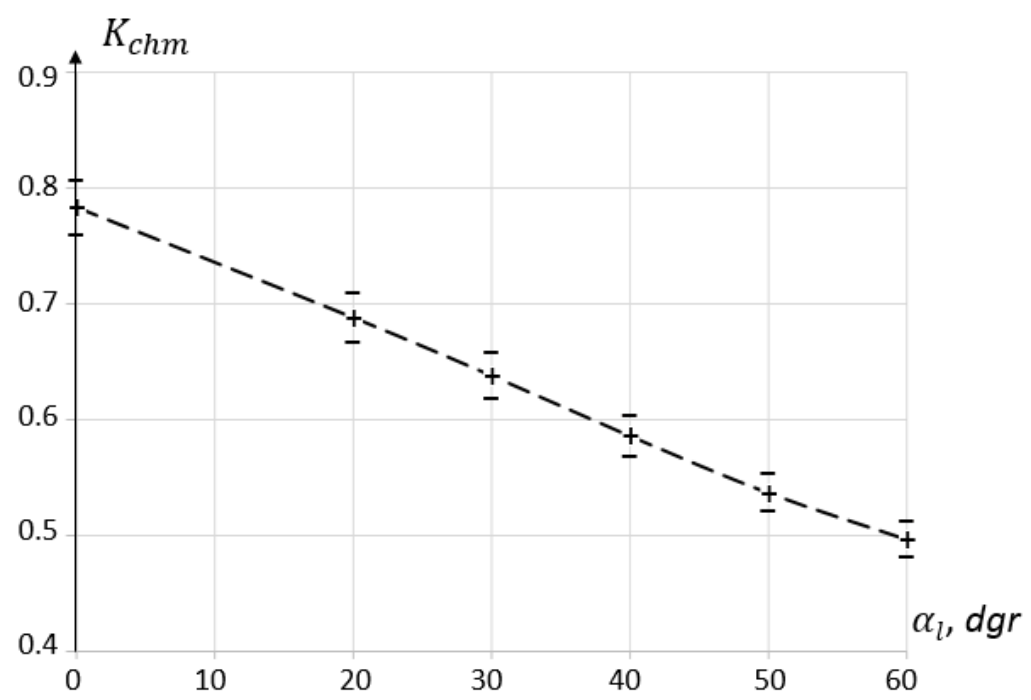

Figure 17. Homogeneity coefficient of mixture $K_{c h m}$ vs. inclination angle of activator blades $\alpha_{l}$ (experimental data).

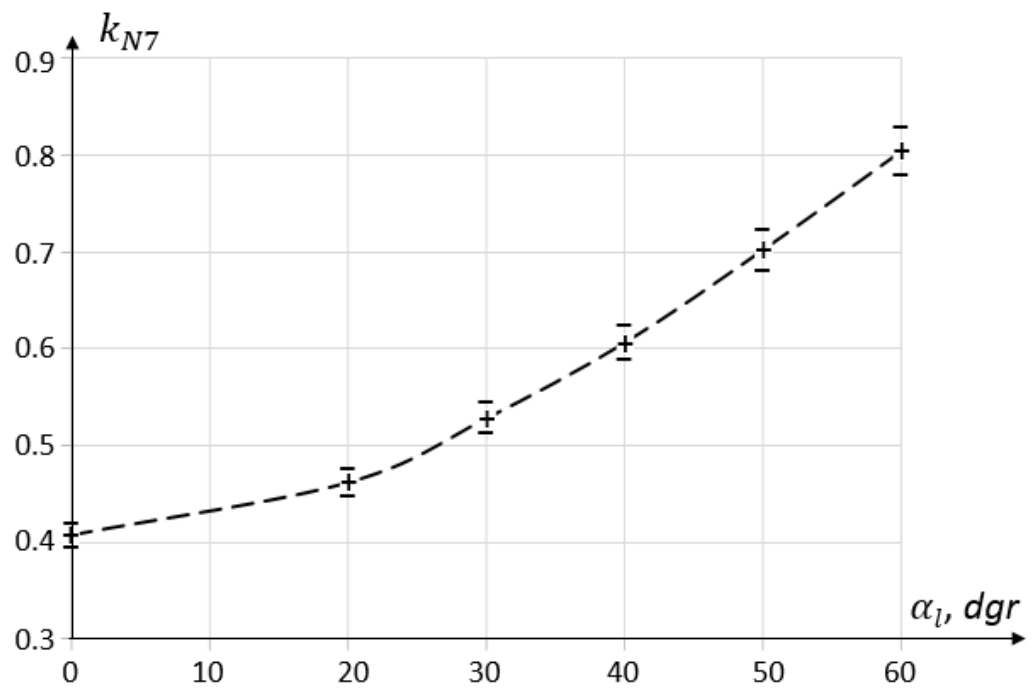

Figure 18. Influence of the angle of inclination $\alpha_{l}$ on the power consumption factor $k_{N 7}$ (experimental data).

From Figure 18, it follows that, in the case when the blades were inclined under an angle $\alpha_{l}=45^{\circ}$ to the rotation plane of mixer, the power consumption coefficient was half in the turbulent flow region compared to the value for similar mixer blades with an angle of inclination $\alpha_{l}=0^{\circ}$. At the same time, the effect on the homogeneity of the foam concrete mixture was minimal. This was confirmed by the results presented in Figure 17.

The obtained experimental data on the influence of the inclination angle of activator blades on the quality of foam concrete mixture and the efficiency of mixing show that the optimal inclination angle of blades was in the range $\alpha_{l}=30-45^{\circ}$.

\section{Conclusions}

Thus, on the basis of the results of the study, the below conclusions can be drawn.

The simplest, most technologically advanced, and most economical activator in operation is a blade activator that operates at a wider range of speeds than propeller and turbine activators and is suitable for mixing foam concrete mixtures with a density from 250 to $1100 \mathrm{~kg} / \mathrm{m}^{3}$. 
The ratio of the height of the foam concrete mixture in the mixer to the mixer diameter $H_{m i x} / D$ is optimal for foam concrete mixtures within values in the range of $0.75-1$.

A high-qualitative (homogeneous throughout the volume) foam concrete mixture with lower energy consumption can be obtained with a $b / D$ ratio in the range of $0.04-0.06$; an increase in the number of reflective partitions leads to an increase in the mixture homogeneity and an increase in power consumption for its preparation. At the same time, an increase in the number of partitions to more than four does not lead to a significant increase in power consumption and an increase in the quality of the mixture. Therefore, it is optimal to install four reflective partitions in the mixer.

The optimal angle of the conical part of mixer $\alpha_{\text {cone }}$ in mixers for the preparation of aerated concrete is from $50^{\circ}$ to $60^{\circ}$.

An increase in the number of blades to three significantly increases the homogeneity of the prepared mixture, while a further increase in the number of blades leads to an insignificant increase in the homogeneity of mixture. Therefore, from the viewpoint of the quality of mixture prepared and from the viewpoint of the manufacturing technology of activator construction, the optimal number of blades is $Z_{m}=3$.

The choice of the ratio of the mixer diameter to the activator diameter should be made taking into account the power consumption and the homogeneity of mixture prepared. Therefore, it was established that the range of optimal values of the parameter $D / d_{a}=4-6$.

The optimal ratio of the length to the width of blade, which ensures the maximum homogeneity of foam concrete mixture at the optimal values of the power factor, is in the range of 0.35-0.45.

The obtained experimental data on the influence of the inclination angle of the activator blades on the quality of foam concrete mixture and the efficiency of mixing show that the optimal angle of the blade inclination is in the range of $30^{\circ}-45^{\circ}$.

Author Contributions: Conceptualization, S.A.S. and E.M.S.; methodology, S.A.S., E.M.S., and A.I.S.; formal analysis, A.I.S., and E.M.S.; investigation, A.Y.P., and S.M.M.; writing-original draft preparation, S.A.S., E.M.S., A.Y.P., and S.M.M.; writing-review and editing, I.A.P. and A.V.C. All authors have read and agreed to the published version of the manuscript.

Funding: This research was funded by the grant from the Ministry of Science and Higher Education of Russia supported by Southern Federal University, grant No. VnGr-07/2020-04-IM.

Acknowledgments: The authors acknowledge the support by the Southern Federal University, grant No. VnGr-07/2020-04-IM (Ministry of Science and Higher Education of Russia).

Conflicts of Interest: The authors declare no conflict of interest.

\section{Abbreviations}

$\begin{array}{ll}\text { HPC } & \text { high-performance concrete } \\ \text { CFD } & \text { computational fluid dynamics } \\ \text { CoV } & \text { coefficient of variation } \\ \text { DEM } & \text { discrete element method } \\ \text { SMI } & \text { subdomain-based mixing index } \\ \text { PC 500 } & \text { Portland cement 500 } \\ \text { FA } & \text { foaming agent } \\ \text { FA1 } & \text { foaming agent No. 1-Penostrom } \\ \text { FA2 } & \text { foaming agent No. 2-Morpen } \\ \text { W/S } & \text { water-solid ratio } \\ \text { SDT } & \text { standard disc turbine } \\ \text { BTD } & \text { blade turbine with downdraft } \\ \text { VTUF } & \text { vane turbine with upward flow }\end{array}$




\section{Nomenclature}

The following nomenclatures are used in this manuscript:

Mathematical parameters

$n_{c r} \quad$ critical speed of mixing the suspension

$\mathrm{rev} / \mathrm{s}$

$N \quad$ power

W

$n_{\text {act }} \quad$ rotation speed of activator

$\mathrm{rev} / \mathrm{s}$

$n, n_{\text {actrpm }}$

rotational speed of activator

rpm

$K_{N}$

dimensionless power criterion

-

$\xi_{m}$

dimensionless resistance coefficient of the mixer

$k_{N}, k_{N I}$,

$k_{N 1}-k_{N 7}$

I

$r$

$\bar{r}$

$v(r)$

$\bar{v}(r)$

dimensionless power consumption factor

corresponding stage of the study

radius of the inner edges of blades

relative average distance from the vertical axis of vehicle

relative average peripheral velocity of stirred medium at a distance $r$ from the vertical axis of apparatus

$\omega_{0} \quad$ angular velocity of the mixer

$\mathrm{rad} / \mathrm{s}$

$N_{0} \quad$ power consumption of the mixer without loading the mixture W

$N_{\text {consum }} \quad$ power, consumed by the mixer for mixing the mixture W

$n_{\text {smp }}$

sample number

$t$ time

$-$

\section{Parameters of the material}

$M_{c r} \quad$ fineness modulus of sand

$d_{c e m} \quad$ average particle diameter of cement and sand

torque applied to blades

$\mu \mathrm{m}$

$M_{t}$

homogeneity coefficient of foam concrete mixture

$\mathrm{Nm}$

$K_{c h m}$

averaged maximum value of density

$\bar{\rho}_{\max }$

averaged minimum value of density

$\mathrm{kg} / \mathrm{m}^{3}$

$\bar{\rho}_{\text {min }}$

medium density

$\frac{\rho}{\rho}$

averaged value of density

\section{Mixer geometrical parameters}

D diameter of apparatus

$H_{\text {mix }}$

height of liquid level in the apparatus

$\mathrm{m}$

mixer vessel

$\mathrm{m}$

$H_{R}$

vertical distance between straight blades

$\mathrm{m}$

vertical distance between first straight blade and the base

$\mathrm{m}$

$H_{R 1}$

$H_{R 2}$

$l$

vertical distance between the second straight blade and the base

$\mathrm{m}$

straight blade width

radius of the inner edges of blades

$\mathrm{m}$

$\mathrm{m}$

$r_{b}$

$\bar{r}_{b}$

$r_{0}$

relative radius of the inner edges of blades

stirrer radius

$\mathrm{m}$

diameter of the straight blades of the mixer

$\mathrm{m}$

$d_{a}$

$h_{1}, h_{2}$

$a_{b l}$

straight blade height

$\mathrm{m}$

length of blades of the mixer

$\mathrm{m}$

width of blades of the mixer

$\mathrm{m}$

$b_{b l}$

inclination angle of the blades

$\mathrm{m}$

$\alpha_{l}$

$B$

$H_{B}$

width of reflective partitions

degree

height of reflective partitions

$\mathrm{m}$

inclination angle of the conical part of the mixer

$\mathrm{m}$

$\alpha_{\text {cone }}$

$Z_{m}$

number of stirrer blades

degree

number of the reflective partitions in the mixer

pcs 


\section{References}

1. Lashchinskii, A.L.; Tolchinskii, A.R. Fundamentals of the Design and Calculation of Chemical Equipment; Mechanical Engineering: Leningrad, Russia, 1970.

2. Vasiltsov, E.A.; Ushakov, V.G. Apparatus for Mixing Liquid Media; Engineering: Leningrad, Russia, 1979.

3. Fadeeva, B.C. The Formation of the Structure of Plastic Pastes of Building Materials During Machine Processing; Stroyizdat: Moscow, Russia, 1972.

4. Wallevik, J.E.; Wallevik, O.H. Analysis of shear rate inside a concrete truck mixer. Cem. Concr. Res. 2017, 95, 9-17. [CrossRef]

5. Lerch, J.O.; Bester, H.L.; Van Rooyen, A.S.; Combrinck, R.; de Villiers, W.I.; Boshoff, W.P. The effect of mixing on the performance of macro synthetic fibre reinforced concrete. Cem. Concr. Res. 2018, 103, 130-139. [CrossRef]

6. Elgindi, T.M.; Zlatoš, A. Universal mixers in all dimensions. Adv. Math. 2019, 356, 106807. [CrossRef]

7. Valigi, M.C.; Logozzo, S.; Rinchi, M. Wear resistance of blades in planetary concrete mixers. Part II: 3D validation of a new mixing blade design and efficiency evaluation. Tribol. Int. 2016, 103, 37-44. [CrossRef]

8. Valigi, M.C.; Logozzo, S.; Rinchi, M. Wear resistance of blades in planetary concrete mixers. Design of a new improved blade shape and 2D validation. Tribol. Int. 2016, 96, 191-201. [CrossRef]

9. Liu, B.; Xu, Z.; Xiao, Q.; Huang, B. Numerical study on solid suspension characteristics of a coaxial mixer in viscous systems. Chin. J. Chem. Eng. 2019, 27, 2325-2336. [CrossRef]

10. Cazacliu, B. In-mixer measurements for describing mixture evolution during concrete mixing. Chem. Eng. Res. Des. 2008, 86, 1423-1433. [CrossRef]

11. Theron, F.; Le Sauze, N. Comparison between three static mixers for emulsification in turbulent flow. Int. J. Multiph. Flow 2011, 37, 488-500. [CrossRef]

12. Haddadi, M.M.; Hosseini, S.H.; Rashtchian, D.; Olazar, M. Comparative analysis of different static mixers performance by CFD technique: An innovative mixer. Chin. J. Chem. Eng. 2020, 28, 672-684. [CrossRef]

13. Cai, R.; Hou, Z.; Zhao, Y. Numerical study on particle mixing in a double-screw conical mixer. Powder Technol. 2019, 352, 193-208. [CrossRef]

14. Mobley, K. Root Cause Failure Analysis; Butterworth-Heinemann: Woburn, MA, USA, 1999. [CrossRef]

15. Harish, V.V.N.; Cho, M.; Shim, J. Effect of Rotating Cylinder on Mixing Performance in a Cylindrical Double-Ribbon Mixer. Appl. Sci. 2019, 9, 5179. [CrossRef]

16. Stel'makh, S.A.; Shcherban, E.M.; Nazhuev, M.P.; Cherpakov, A.V.; Parinov, I.A. Experimental and Theoretical Approach to Assessing the Strength Properties of Layered Tubular Constructions Made of Heavy Concrete. In Physics, Mechanics of New Materials and Their Applications, Proceedings of the 2019 International Conference, Hanoi, Vietnam, 7-10 November 2019; Nova Science Publishers: New York, NY, USA, 2020; pp. 291-298. ISBN 978-1-53618-255-2.

17. Cherpakov, A.; Egorochkina, I.; Shlyakhova, E.; Kharitonov, A.; Zarovny, A.; Dobrohodskaya, S. Using technique vibration diagnostics for assessing the quality of power transmission line supports repairs. Matec Web Conf. 2017, 106, 04009. [CrossRef]

18. Cherpakov, A.V.; Soloviev, A.N.; Gricenko, V.V.; Goncharov, O.U. Damages identification in the cantilever-based on the parameters of the natural oscillations. Def. Sci. J. 2016, 66, 44-50. [CrossRef]

19. Morgun, L.V.; Morgun, V.N. The effect of dispersed reinforcement on the aggregate stability of foam concrete mixtures. Build. Mater. 2003, 1, 33-35.

20. Rakhimbaev, S.M.; Tverdokhlebov, D.V.; Tarasenko, V.N. Comparative studies of the rheological properties of foam concrete mixtures with foam concentrates "Penostrom" and "Neopor". Build. Mater. 2005, 6, 64-67.

21. Mal'tsev, E.V.; Kozlov, A.V.; Kaklyugin, A.V.; Kozlov, G.A. Aerated concrete based on ash microspheres of Novocherkasskaya TPP. Bull. Bstu Named V.G. Shukhov. 2003, 5, 404-405.

22. Braginsky, L.N.; Begachev, V.I.; Barabash, V.M. Stirring in Liquid Media; Chemistry: Leningrad, Russia, 1984.

23. Rumyantsev, B.M.; Kritarasov, D.S. Production and use of foam gypsum materials. Build. Mat. Equip. Technol. XXI Century 2004, 9, 74-75.

24. Strenk, F. Stirring and Agitator Apparatus; Chemistry: Leningrad, Russia, 1975.

25. Torlina, E.A.; Shuiskii, A.I.; Yazyeva, S.B.; Tkachenko, G.A. Activation of cement paste and aerated concrete mixture in electromagnetic grinding units. Eng. J. Don. 2011, 2. 
26. Pavlov, A.N.; Gol'tsov, Y.I.; Mailyan, L.R.; Shcherban', E.M.; Stel'makh, S.A. Relaxation processes during activation of cement mixing water. IOP Conf. Ser. Mater. Sci. Eng. 2020, 896, 012124. [CrossRef]

27. Shuisky, A.; Stelmakh, S.; Shcherban, E.; Torlina, E. Recipe-technological aspects of improving the properties of nonautoclaved aerated concrete. Matec. Web Conf. 2017, 129, 05011. [CrossRef]

28. Shcherban', E.M.; Gol'tsov, Y.I.; Tkachenko, G.A.; Stel'makh, S.A. Prescription and technological factors and their role in the formation of the properties of foam concrete obtained from mixtures treated with an alternating electric field. Eng. J. Don. 2012, 3.

29. Stel'makh, S.A.; Shcherban', E.M.; Zholobov, A.L. Electroactivation of Foam Concrete for Buildings and Structures with Improved Constructive and Energy Efficient Characteristics. IOP Conf. Ser. Mater. Sci. Eng. 2018, 463, 022034. [CrossRef]

Publisher's Note: MDPI stays neutral with regard to jurisdictional claims in published maps and institutional affiliations.

(C) 2020 by the authors. Licensee MDPI, Basel, Switzerland. This article is an open access article distributed under the terms and conditions of the Creative Commons Attribution (CC BY) license (http://creativecommons.org/licenses/by/4.0/). 